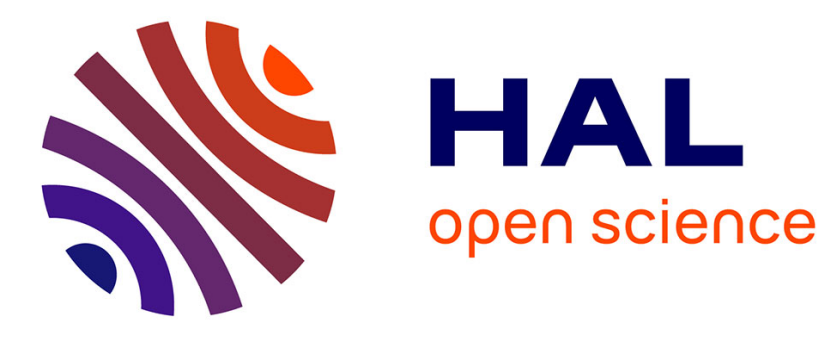

\title{
Using voxels in the simulation of manufacturing processes
}

\author{
Anthony Surleraux, Samuel Bigot, Jean-Philippe Pernot, Gianluca d'Urso, \\ Cristina Merla
}

\section{- To cite this version:}

Anthony Surleraux, Samuel Bigot, Jean-Philippe Pernot, Gianluca d'Urso, Cristina Merla. Using voxels in the simulation of manufacturing processes. International Conference on Multi-Material Micro Manufacture (11; 2015;Milan), 2015, Milan, Italy. pp.1-5. hal-01410033

\section{HAL Id: hal-01410033 \\ https://hal.science/hal-01410033}

Submitted on 6 Dec 2016

HAL is a multi-disciplinary open access archive for the deposit and dissemination of scientific research documents, whether they are published or not. The documents may come from teaching and research institutions in France or abroad, or from public or private research centers.
L'archive ouverte pluridisciplinaire HAL, est destinée au dépôt et à la diffusion de documents scientifiques de niveau recherche, publiés ou non, émanant des établissements d'enseignement et de recherche français ou étrangers, des laboratoires publics ou privés. 


\title{
Using voxels in the simulation of manufacturing processes
}

\author{
A. Surleraux ${ }^{1}$, S. Bigot ${ }^{1}$, J-P. Pernot ${ }^{2}$, G. D’Urso ${ }^{3}$, C.Merla $^{3}$ \\ ${ }^{1}$ Manufacturing Engineering Centre, Cardiff University, Cardiff, UK. E-mail: SurlerauxAB@cardiff.ac.uk \\ ${ }^{2}$ Arts et Métiers ParisTech, LSIS UMR CNRS 7296, Aix-en-Provence, France. \\ ${ }^{3}$ Università degli Studi di Bergamo, Dip. di Ingegneria Gestionale, dell'Informazione e della Produzione, BG, Italy.
}

\begin{abstract}
The present paper introduces the use of voxels embedded in an octree structure in order to numerically simulate manufacturing processes. In particular, micro electrical discharge machining ( $\mu E D M)$ is used here as a case study. The involved elements (tool and workpiece) are modelized in a volumetric manner using voxels and the process is simulated on a step-by step basis. Comparisons using the Hausdorff metric with experimental results are included and discussed.
\end{abstract}

Keywords: micro, EDM, voxel, simulation, octree.

\section{Introduction}

Electrical Discharge Machining (EDM) is a manufacturing process where material removal is due to successive electrical discharges. Its main interest lies in the possibility of machining any conductive material regardless of hardness.

A few variants of EDM are in use but share an underlying principle: the tool and the workpiece (or electrodes) are immerged in a dielectric fluid and submitted to an electrical current. The gap between them is reduced until the dielectric reaches its breakdown voltage. The current is then free to flow from one electrode to the other creating a plasma channel. The energy dissipated partly evaporates the tool and workpiece leading to the formation of craters.

Micro-EDM $(\mu E D M)$ deals with micrometric dimensions. The polarity is usually inverted in order to alleviate the effects of tool wear: as the process goes on, material is removed from the tool, modifying its shape. While of manageable consequences in classic EDM, the influence of tool wear is most notable in its micro counterpart.

While proven methods exist for $\mu$ EDM milling $[1,2$, 3] to compensate for the tool wear, die-sinking $\mu$ EDM would require the use of multiple tools to obtain the desired geometries.

The ability to predict the location and intensity of wear in die-sinking $\mu \mathrm{EDM}$ would enable the design of optimal tools able to compensate for the upcoming wear and reduce the number of electrodes required for a successful machining.

Until now most modelling works that have been conducted focus on the development of single crater (or single spark) models in which physical equations are used under certain assumptions to determine the shape of the resulting craters of a single spark. A few $[4,5,6]$ have proposed simulation methods of micro-EDM usually using a grid of points to model the involved geometries. More recently, a method involving z-maps [7] has been developed. However there is an inherent limitation in the use of height maps which can't represent overhangs without resorting to additional techniques which isn't present with voxels.

Previous research [8] focused on the use of NURBS to model the surface of micro-EDM electrodes during the process but proved to be slow and prone to errors in the case of long simulations.

Against this background, the aim is to develop a new approach for the accurate and fast modeling of deformation affecting complex 3D shaped micro electrodes. More specifically the present research has the following objectives:

- Design a simulation tool to simulate a complete diesinking micro-EDM process using voxels (volumetric pixels) as the support geometries.

- Assess the viability of this method.

\section{Voxels and octrees}

Voxel stands short for "volumetric pixel" and, as the name implies, can be considered as being the threedimensional equivalent of pixels. As mentioned before, they are used for the representation of threedimensional elements. The most common approach is to set them in a uniform grid aligned with the coordinate axes. Although recent developments have increased their use in the computer graphics industry, their discrete nature has made them unsuitable for the representation of detailed scenes. Consequently, voxels have mostly been used in the visualization of volumetric data such as in the medical imagery field. It is of note that voxels aren't of a widespread use in the manufacturing field. A use of them can be found in the simulation of multi-axis CNC machining [9].

Octrees are tree data structures in which each node can be subdivided into eight children hence the name. The main node is designated as the root and a non-subdivided node is called a leaf node. The use of voxels for the volumetric representation of objects can be quite memory-consuming if a decent resolution is desired. Embedding voxels in an octree data structure drastically reduces the number of voxels required to represent said object: large full sections don't need to 
be subdivided into smaller nodes. In addition, their hierarchical nature are well suited for parallelization.

\section{A numerical simulation tool}

\subsection{Overview}

As explained in section 1, $\mu$ EDM involves two main elements: the tool and the workpiece. What is proposed in the present paper is to model both of those electrodes with voxels embedded in a pointer-based octree and use those volumetric representations in order to simulate the process.

The simulation is an iterative process that ends when the desired tool movement is achieved. In diesinking micro-EDM this usually consists in a simple vertical movement from the tool in order to reach a specific depth called the objective depth, $D_{o b j}$

An iteration firstly consists in determining the locations of the sparks. It is assumed here that the spark takes the path of least resistance and therefore occurs at the minimum distance between both geometries.

The found minimum distance is then compared to a value called the machining gap, $\mathrm{M}_{\mathrm{g}}$ to determine if a spark can occur. In the case where it is possible for a discharge to happen, a crater is added to each of the electrodes via the removal of predetermined volumes $V_{t}$ and $V_{w}$ on the tool and the workpiece.

If no spark can take place, the tool is moved down with an increment of $\Delta_{z}$

The process is repeated until a total vertical movement $D_{o b j}$ is achieved. Two main sub-algorithms can be identified and are further detailed in the following sections.

\subsection{Distance computations}

During the simulation of the micro-EDM process, the electrical discharge is supposed to take place at the smallest distance between both electrodes.

This section deals with the search of the minimal distance between two disjoint octrees. In addition, both of those are axis-aligned. The presented method is based on [10] with the addition of some fast exit conditions. The pseudocode is as follows:

MinimumDistance(tool, workpiece, levels, gap)

current $=0$, done $=$ false

candidates $=$ toolRoot, workpieceRoot

smallestMaximum = upperDistanceBound(tool, Root)

if(lowerDistanceBound(tool, workpiece) > gap) return null

while(!done \&\& current < levels) nextCandidates $=$ Candidates (candidates,

levels, gap, ref smallestMaximum, ref done) if(nextCandidates $==$ null) return null candidates $=$ nextCandidates current++

end while

return nextCandidates

end

Candidates(candidates, levels, gap, ref

smallestMaximum, ref done)

foreach(couple in candidates) output $=$ null

if(coupleAreLeaves \&\& lowerBound

$<$ gap) return couple

foreach(children1 of couple.item1)

foreach(children2 of couple.item2)

if(maximum $<$ machiningGap)

done $=$ true

output.Clear

output.Add(children1, children2)

return output

if(upperBound(children1, children2) <

smallestMaximum)

smallestMaximum = upperBound $($ children 1 , children2)

if(minimum <= smallestMaximum \&\& minimum

return output output.Add(children1, children2)

Several other minimization algorithms have been tested and the results tabulated in table 1.

Table 1

Computation times and found distances for several optimization methods.

\begin{tabular}{lllll}
\hline Method & Octree & PSO & DE & MOL \\
\hline Avg time $(\mathrm{ms})$ & 3.25 & 23.96 & 102.60 & 75.47 \\
Avg distance $(\mu \mathrm{m})$ & 19.95 & 18.54 & 19.12 & 18.12 \\
\hline
\end{tabular}

The algorithms were all tested on the same scenario, the machining of a $100 \mu \mathrm{m}$ spherical hole with a gap of $20 \mu \mathrm{m}$. The particle swarm optimization, differential evolution and many optimizing links methods were previously optimized through meta-optimization. Genetic algorithms were tested since they are commonly used for the search of a minimum distance between potentially concave objects. The octree hierarchical search method (13 levels) severely outperforms the others. While the average distance found is higher than the others, it is indicative of the fact that genetic algorithms have a hard time finding the boundaries of the volumes due to their complexity and require the tool to be often prematurely moved down. The gradient descent was tested but often lead to the intersection of the volumes due to repeated local minima being found.

\subsection{Volume removal}

The locations of the discharge determined, the simulation must now proceed to the removal of voxels in order to mimic the presence of a crater.
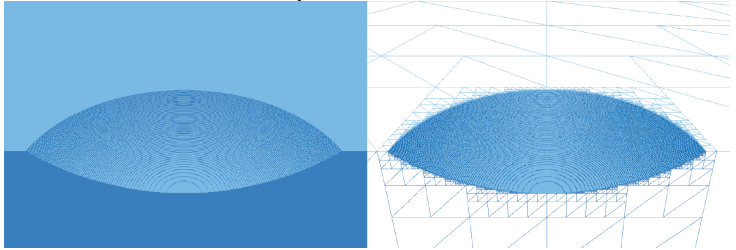

Fig.1. Single crater at a resolution of one voxel per $125 \mathrm{~nm}$.

It is hypothesized that each spark removes the same volume $V_{t}$ and $V_{w}$ on the tool and the workpiece.

Moreover, the material removal process must subtract the volume in a manner that is identifiable as a crater. 
The literature suggests that the shape of the craters resulting from the micro-EDM process is ellipsoidal in nature [11]. While possible to implement, the use of a sphere as an approximation of an ellipsoid yields acceptable results with increased speed. A crater is considered here as a spherical cap of radius $\mathrm{Cr}_{i}$ and depth $c d_{i}$ of a volume $V c_{i}$ (where $i=t$ for the tool and $i=w$ for the workpiece).

$$
V c_{i}=\pi c d_{i}\left(2 c r_{i}^{2}+c d_{i}^{2}\right) / 6
$$

The support sphere radius is:

$$
R_{i}=\left(c d_{i}^{2}+c r_{i}^{2}\right) /\left(2 c d_{i}^{2}\right)
$$

Similarly to the distance algorithm, the volume removal algorithm searches the octrees in a top-down manner keeping only nodes that intersect with the sphere and deleting those that are inside. The intersection test is made by finding the smallest cubed distance $d$ between a cube and a point and comparing it to $R_{i}^{2}$. The intersection test returns true if:

$$
d \leq R_{i}^{2}
$$

\section{Experimental validation}

\subsection{Overview}

In order to validate the numerical simulation tool presented in the previous sections as well as finetuning its parameters, an experimental validation process needs to be conducted. In order to achieve this, two different tool shapes have been devised as can be seen in figure 2 and realized using wire-EDM. The spherical tool has a diameter of $246 \mu \mathrm{m}$ while the triangular tool has a side size of $64 \mu \mathrm{m}$. Before being used in a machining process, those tools have been measured with the use of a micro-tomograph of a resolution of a micron [12, 13]. This measurement methodology enables the modelling of the physical tools as three-dimensional meshes that can be exported in the simulation tool and converted as voxels embedded in an octree data structure using an adapted method from [14]

A comparison of a three-dimensional mesh and the resulting solid voxelisation can be observed in figure 2 . Using the same measurement method, the tools as well as the holes are measured again after the machining process and subsequently compared to the simulation results.

\subsection{Experimental parameters and results}

The experiments were conducted on a Sarix SX200 micro-EDM machine using tungsten carbide as a material for the tool and aluminium for the workpiece. Two features were machined with the machining parameters in table 2 .

Those parameters were chosen in respect with the dimensions of the features to be machined as well as to ease the corresponding simulations. At the present hour, simulating the removal of small craters (dimensions smaller than a micron) dramatically increases the required voxel resolution for the simulated craters to have a clearly defined shape and consequently the memory usage and computation time.
A depth of $100 \mu \mathrm{m}$ is set for the machining operations across all four tools. This depth was selected in order to provide a noticeable wear on the tool without completely destroying the features on it.
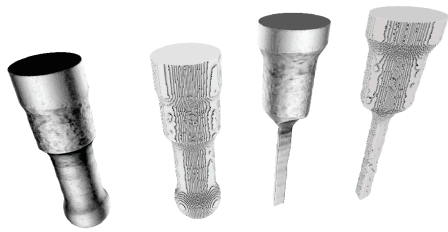

Fig. 2. Meshed models of the tools and their voxelized counterparts at a resolution of 0.5 voxel per micron.

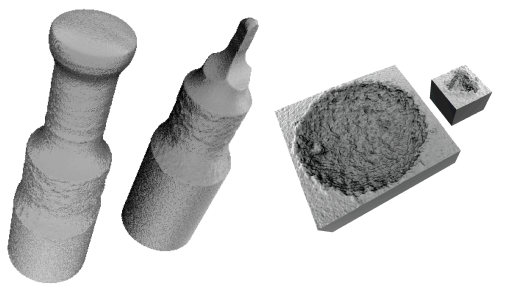

Fig. 3. Tool and features obtained after machining.
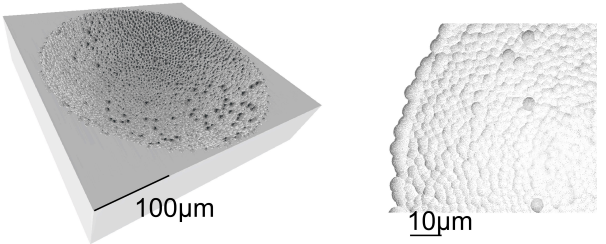

Fig. 4. Spherical feature obtained by simulation (left) and detail of the craters (right).

Experimental results are visible in figure 4.

Table 2

Experimental parameters. Unitless parameters are machine index values.

\begin{tabular}{ll}
\hline Parameter & Value \\
\hline Energy & 200 \\
Current & 20 \\
Voltage $(\mathrm{V})$ & 90 \\
Frequency $(\mathrm{Hz})$ & 100 \\
Width $(\mathrm{ms})$ & 5 \\
\hline
\end{tabular}

Table 3

Simulation parameters.

\begin{tabular}{ll}
\hline Parameter & Value \\
\hline Tool crater radius $(\mu \mathrm{m})$ & 6.20 \\
Tool crater depth $(\mu \mathrm{m})$ & 4.39 \\
Workpiece crater radius $(\mu \mathrm{m})$ & 6.65 \\
Workpiece crater depth $(\mu \mathrm{m})$ & 4.42 \\
Machining gap $(\mu \mathrm{m})$ & 3.01 \\
Objective depth $(\mu \mathrm{m})$ & 100 \\
\hline
\end{tabular}

\subsection{Simulation parameters and results}

The crater geometrical information in table 3 was 
obtained from the experimental results, directly on the $3 \mathrm{D}$ meshes. Contrarily to what is seen in figure 2 , only a part of the tools were voxelized in order to achieve a

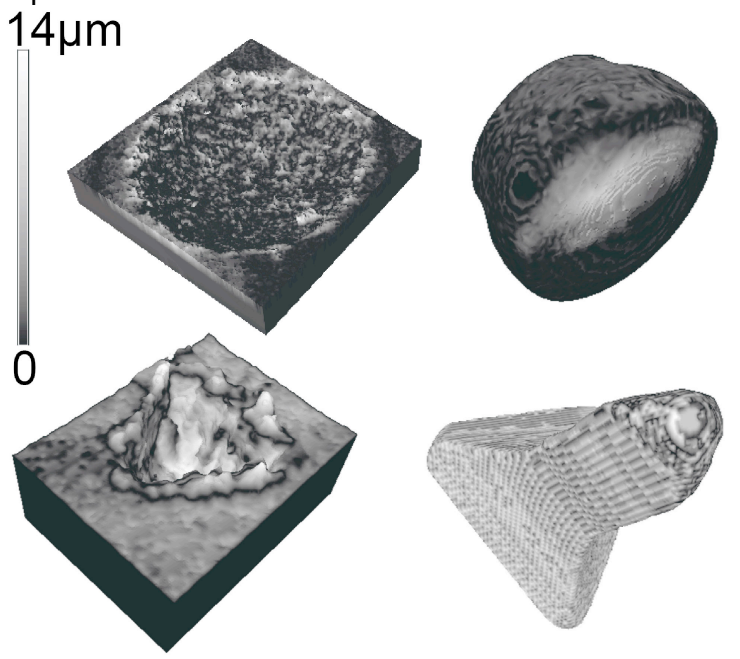

Fig. 5. Hausdorff distance maps

higher resolution of $1 \mathrm{voxel} / \mathrm{micron}$. The simulation was performed on a PC with a core i5 processor at $3.4 \mathrm{Ghz}$ and lasted 2 hours and 12 minutes.

\subsection{Experimental and simulation comparison}

The comparison of experimental and simulated results is made by using the voxel octrees of the electrodes after machining and after the simulation.

A tool designed to compare meshes, Metro [15], was used to compute the Hausdorff distance between two meshes. The Hausdorff distance between two sets of points $X$ and $Y$ is:

$$
d_{H}(X, Y)=\max \left\{\sup _{x \in X} \inf _{y \in Y} d(x, y), \sup _{y \in Y} \inf _{x \in X} d(x, y)\right\}
$$

Where $d$ is the Euclidian distance function in the present case. The results of the Hausdorff filter are tabulated in table 4 and a visual representation of the meshes' differences is visible in figure 5 .

Table 4

Hausdorff distances. (WP: Workpiece).

\begin{tabular}{lllll}
\cline { 2 - 5 } & \multicolumn{2}{c}{ Sphere } & \multicolumn{2}{c}{ Triangle } \\
\cline { 2 - 5 } & WP & Tool & WP & Tool \\
\hline$d_{H} \min (\mu \mathrm{m})$ & 0.00 & 0.00 & 0.00 & 0.00 \\
$d_{H} \max (\mu \mathrm{m})$ & 12.86 & 8.59 & 8.44 & 2.39 \\
$d_{H} \operatorname{mean}(\mu \mathrm{m})$ & 0.84 & 1.13 & 0.73 & 0.86 \\
$d_{H} \operatorname{RMS}(\mu \mathrm{m})$ & 1.53 & 1.61 & 1.48 & 0.99
\end{tabular}

\section{Conclusion and future works}

Considering the variability of the micro-EDM process, the accuracy offered by the presented method can be concluded as being good with a Hausdorff distance RMS value under two microns. Some big local differences are noticeable and are due to re-solidified debris that the simulation does not account for.

In order to generalize the present work and not limit it to a specific combination of materials machined on a specific machine, direct data collection of spark energies is required in order to build a database and predictive methods to be used to feed the simulation tool with the correct parameters.

\section{Acknowledgements}

This work was supported by the Engineering and Physical Sciences Research Council [ EP/J004901/1] and made possible thanks to italian project funds. The authors are grateful to Prof. Maurizio Santini for the micro-tomography analysis.

\section{References}

[1] Z. Yu et al, "Micro-EDM for ThreeDimensional Cavities Development of Uniform Wear Method", Annals of the CIRP, 1998; 47/1: pp.169172.

[2] T. Yuzawa et al, "Micro Electric Discharge Scanning Using a Mini-Size Cylindrical Electrode", Kata Gijutsu, 1997; 12/8: pp.104-105.

[3] P. Bleys et al, "Real-time Tool Wear Compensation in Milling EDM", Annals of the CIRP, 2002; 51/1: pp.157-160.

[4] S. Bigot et al, "A New Modelling Framework for Die-Sinking Micro EDM", Proceedings of the $9^{\text {th }}$ International Conference on Multi-Material Manufacture: 2012, pp.51-55.

[5] C. Tricarico et al, "Geometrical Simulation of the Die-Sinking Process.", Annals of the CIRP, 1988; 37/1: pp.191-196.

[6] S. Heo et al, "Virtual EDM Simulator: ThreeDimensional Geometric Simulation of Micro-EDM Milling Processes", International Journal of Machine Tools and Manufacture, 2009; 49/12-13: pp.1029-1034. [7] S. Heo et al, "Geometric Simulation Based Tool Wear Compensation in Micro Die-Sinking EDM Process", Poster presented at the $29^{\text {th }}$ Annual Meeting of the American Society for Precision Engineering, 2014, Boston, MA.

[8] S. Bigot et al, "Micro-EDM numerical simulation and experimental validation", Proceedings of the $10^{\text {th }}$ International Conference on Multi-Material Manufacture: 2013, pp.414-417.

[9] D. Jang et al, "Voxel-Based Virtual Multi-Axis machining", International Journal of Manufacturing Technology, 2000; 16: pp.709-713.

[10] A. Boorman et al, "An Iterative, Octree-Based Algorithm for Distance Computation Between Polyhedra with Complex Surfaces", Proceedings of the International ASCE Workshop on Computing in Civil Engineering: 2007, pp.103-110.

[11] J. Tao et al, "Modeling of the Anode Crater formation in Electrical Discharge Machining", Journal of Manufacturing Science and Engineering, 2012; 134:011002-1.

[12] M. Santini et al, "X-ray computed microtomography for drop shape analysis and contact angle measurement", J. Colloid Interface Sci., 2013; 409: pp.204-210.

[13] M. Santini et al, "3D X-ray Micro Computed Tomography on Multiphase Drop Interfaces: From Biomimetic to Functional Applications", Colloids Interface Sci. Commu., 2014; 1:pp.14-17.

[14] M. Schwarz et al, "Fast Parallel Surface and Solid Voxelization on GPUs", ACM Transactions on Graphics, 2010; 29/6 (Proceedings of SIGGRAPH Asia 
2010): 179 .

[15] P. Cignoni et al, "Metro: Measuring Error on

Simplified Surfaces", Computer Graphics Forum, 1998;

17/2, pp.167-174. 